International Journal on Cybernetics \& Informatics (IJCI) Vol. 3, No. 4, August 2014

\title{
Optimal control Problem for Processes Represented By Stochastic Sequential MaCHINE
}

\author{
Yakup H. HACI and Muhammet CANDAN \\ Department of Mathematics, Canakkale Onsekiz Mart University, Canakkale, Turkey
}

\begin{abstract}
In this paper, optimal control problem for processes represented by stochastic sequential machine is analyzed. Principle of optimality is proven for the considered problem. Then by using method of dynamical programming, solution of optimal control problem is found.
\end{abstract}

\section{Keywords}

Optimal control problem, stochastic sequential machine, dynamical programming.

\section{INTRODUCTION}

Stochastic sequential machine (SSM) is the one of the most developing field of discrete system theory [2], [3]. It plays an important role in many areas such as construction of finite dynamical system, imitation modelling problem, coding of discrete systems and identification problems. Thus, it points out that it requires a comprehensive research.

\section{SSM}

SSM is generalization of multi-parametric finite sequential machine[3] but it contains probability variable. General form of this system is defined by[6]:

$$
K=<X, S, Y, s^{0}, p(\omega), F_{v}(.), G>\quad v=1,2, \ldots k
$$

where $X=[G F(2)]^{r}, S=[G F(2)]^{m}$ and $Y=[G F(2)]^{q}$ are input, state and output index (alphabet) respectively; $s^{0}$ is initial state vector, $p(\omega)$ is determitistic discrete probability distribution $\left(\Omega=\left\{\omega_{1}, \omega_{2}, \ldots, \omega_{p}\right\}\right.$ is finite set, $\left.p(\omega)=\left\{p\left(\omega_{i}\right): \omega_{i} \in \Omega, \sum_{\omega_{i} \in \Omega} p\left(\omega_{i}\right)=1\right\}\right)$, characteristic Boolean vector functions [7] denoted by $F_{v}(\cdot)=\left\{F_{v_{1}}(\cdot), \ldots, F_{v_{m}}(\cdot)\right\}$ which are also known as transfer functions are nonlinear functions defined on the set $Z^{k} \times[G F(2)]^{m} \times[G F(2)]^{r}$ and $G$ are an output characteristic functions defined on $G F(2)$ where $G F(2)$ is Galois field[8] and the symbol (.) denotes $(c, s(c), x(c))$ for simplicity.

In addition to the definition, SSM is represented by:

$$
s\left(c+e_{v}\right)=F_{v}(c, s(c), x(c), \omega(c)) v=1,2, \ldots k
$$


International Journal on Cybernetics \& Informatics (IJCI) Vol. 3, No. 4, August 2014

$$
y(c)=G(s(c))
$$

where $s(c), x(c), y(c)$ are $m, r$ and $q$ dimensional state, input and output vectors at the point

$c$ respectively, $\omega(c)$ is a random variable [6], $c=\left\{c \mid c \in Z^{k}, c_{1}^{0} \leq c_{1} \leq c_{1}^{L_{1}}, \ldots, c_{k}^{0} \leq c_{k} \leq c_{k}^{L_{k}}, c_{i} \in Z\right\}$

is point in $Z^{k}$, determining position $L_{i},(i=1,2, \ldots k$ positive integer $)$ is the duration of the stage $i$ of this process. $Z$ is set of integers and $e_{v}=(0, \ldots, 0,1,0, \ldots, 0)$.

In SSM, each random variable $\omega$ has a special case. For instance, $\omega$ is an input variable in the identification problem of the SSM. However, $\omega$ is a set of all possible states in the synthesis of optimal sequential machine

Moreover, the state of the system depends on the random variable $\omega$ which affects not only parameters of the SSM but also the input variable.

Finally, equation (2) is transformed to:

$$
s\left(c+e_{v}\right)=F_{v}(c, s(c), x(c) \oplus \omega(c)) \quad v=1,2, \ldots, k
$$

where symbol $\oplus$ means that $\mathrm{x} \oplus \mathrm{w}$ is always in input alphabet $X$.

The discrete optimal processes given by SSM are characterized by functional: $\bar{J}(x)=M_{\omega}\left\{\phi\left(s\left(c^{L}\right)\right)\right\}$

where $M_{\omega}\{$.$\} is a mathematical expected value of \omega$.

\section{Optimal Control Problem And Principle Of Optimality}

We can state optimal control problem [2] for processes represented by SSM as below: In order for given SSM to start from the known inital state $s^{0}$ to go any desired state $s^{*}\left(c^{L}\right)$, to which we expect to access in $L$ steps, a control $x(c) \in X$ [4] must exist such that the functional in (1) has a minimal value:

$$
\begin{aligned}
& s\left(c+e_{v}\right)=\hat{F}_{v}(c, s(c), x(c), \omega(c)), c \in G_{d} \quad v=1,2, \ldots k \\
& s\left(c^{0}\right)=s^{0}, \quad x(c) \in X, c \in G_{d} \\
& \hat{F}_{v}\left(c+e_{\mu}, \hat{F}_{\mu}(c, s(c), x(c)), x\left(c+e_{\mu}\right), \omega\left(c+e_{\mu}\right)\right)=\hat{F}_{\mu}\left(c+e_{v} \hat{F}_{v}(c, s(c), x(c)), x\left(c+e_{v}\right) \omega\left(c+e_{v}\right)\right.
\end{aligned}
$$

$\bar{J}(x)=M_{\omega}\left\{\phi\left(s\left(c^{L}\right)\right\} \rightarrow \min \right.$

where $\hat{F}_{v}() \quad.(v=1, \ldots, k)$ denotes the pseudo Boolean expression of the Boolean vector function[7] $\hat{F}().(v=1, \ldots, k)$ and $L=L_{1}+L_{2}+\ldots+L_{k}$ is the time duration of this process.

It is well-known that method of dynamic programming [5] is used for solution of optimal control problem. If we make use of this method to solve the considered problem then, (6)-(9) can be formulate as an optimal problem:

$s\left(c+e_{v}\right)=\hat{F}_{v}(c, s(c), x(c), \omega(c)), c \in G_{d}(\sigma)$ 
International Journal on Cybernetics \& Informatics (IJCI) Vol. 3, No. 4, August 2014

$s(\sigma)=\aleph$

$x(c) \in X, c \in G_{d}(\sigma)$

$\hat{F}_{v}\left(c+e_{\mu}, \hat{F}_{\mu}(c, s(c), x(c), \omega(c)), x\left(c+e_{\mu}\right), \omega\left(c+e_{\mu}\right)\right)=\hat{F}_{\mu}\left(c+e_{v}, \hat{F}_{v}(c, s(c), x(c), \omega(c)), x\left(c+e_{v}\right), \omega\left(c+e_{v}\right)\right)$

$\bar{J}(x)=M_{\omega}\left\{\phi\left(s\left(c^{L}\right)\right)\right\} \rightarrow \min$

where $\boldsymbol{\aleph}$ is an arbitrary element in $S$. As it can be seen from (10)-(14), if we substitute $\sigma=c^{0}$ or $\boldsymbol{\aleph}=s^{0}$ into problem (10)-(14) we obtain first problem stated above. If the conditions for existence of unique solution are satisfied then for the given initial condition $s(\sigma)=\boldsymbol{N}$ and given $x(c)\left(c \in G_{d}(\sigma)\right)$, we find a unique $s(c)$. That is, the functional (14) is the function of the parameters $\boldsymbol{\aleph}$ and $x(c)\left(c \in G_{d}(\sigma)\right)$ :

$\bar{J}(x)=\bar{J}\left(\aleph, x\left(G_{d}(\sigma)\right)\right.$

where $x\left(G_{d}(\sigma)\right)$ denotes the range of the control $x(c)$ on the points $c \in G_{d}(\sigma)$ :

$x\left(G_{d}(\sigma)\right)=\left\{x(c), c \in G_{d}(\sigma)\right\}$

from the unique solution condition of the system (6), we find that the stochastic process can be investigated in the set $G_{d}(\sigma)$ and also in the set

$G_{d_{1}}(\sigma)=\left\{c ; c_{1}^{0} \leq c_{1}<\sigma_{1}, \ldots, c_{k}^{0} \leq c_{k}<\sigma_{k}\right\}$

Definition. We say that the control $x(c),\left(c \in G_{d}(\sigma)\right)$ which minimizes the functional (5) in the problem (10)-(14) is optimal control with respect to the initial pair $(\sigma, \aleph)$ on the region $G_{d}(\sigma)$

Suppose that $x^{0}(c)$ is an optimal control with respect to the initial pair $\left(c^{0}, s^{0}\right)$ on the region $G_{d}$ and $s^{0}(c)$ is admissible optimal trajectory. Then $x^{0}(c)$ is an optimal control with respect to the initial pair $\left(\sigma, s^{0}(\sigma)\right)$ on the region $G_{d}(\sigma)$ for every $G_{d}$.

Proof. Suppose the contrary. Then there exists $x(c) \in X, c \in G_{d}(\sigma)$ such that we have $\bar{J}\left(\aleph, x\left(G_{d}(\sigma)\right)<\bar{J}\left(\aleph, x^{0}\left(G_{d}(\sigma)\right)\right.\right.$

We choose a new control process $\tilde{x}(c), c \in G_{d}$ as follows:

$\tilde{x}(c)=\left\{\begin{array}{c}x^{0}(c), c \in G_{d_{1}}(\sigma) \\ x(c), c \in G_{d}(\sigma)\end{array}\right.$

As it can be seen, (19) is an admissible control such that 
$\bar{J}\left(s^{0}, \tilde{x}\left(G_{d}\right)\right)=\bar{J}\left(s^{0}, \tilde{x}\left(G_{d_{1}}(\sigma) \cup G_{d}(\sigma)\right)\right.$.

According to the condition, $s^{0}(\sigma)=\aleph$. Thus we have

$\bar{J}\left(s^{0}, \tilde{x}\left(G_{d_{1}}(\sigma) \cup G_{d}(\sigma)\right)=\bar{J}\left(s^{0}(\sigma), \tilde{x}\left(G_{d}(\sigma)\right)==\bar{J}\left(\aleph, x\left(G_{d}(\sigma)\right)\right)<\bar{J}\left(\aleph, x^{0}\left(G_{d}(\sigma)\right)\right)=\right.\right.$

$=\bar{J}\left(s(\sigma), x^{0}\left(G_{d}(\sigma)\right)\right)=\bar{J}\left(s^{0}, x^{0}\left(G_{d}\right)\right)$

and by virtue of (2.10) and (2.11) we can obtain

$\bar{J}\left(s^{0}, \tilde{x}\left(G_{d}\right)\right)<\bar{J}\left(s^{0}, x^{0}\left(G_{d}\right)\right)$

Hence, (22) contradicts the hypothesis that the control $x^{0}(c), c \in G_{d}$ is optimal. This completes the proof of the theorem.

Let a function (for every fixed $\sigma$ and $\boldsymbol{N}$ ) be corresponding to the optimal value of pseudo Boolean functional in the problem (10)-(14):

$B(\sigma, \aleph)=\min M_{\omega}\left\{\phi\left(s\left(c^{L}\right)\right)\right\}$.

where minimization on the set of admissible control $x(c), c \in G_{d}(\sigma)$.

Now, we determine method of dynamical programming(It is known as Bellman equation) [5] for $B(\sigma, \aleph)$. Suppose that $x^{0}(c), c \in G_{d}$ is the admissible control corresponding to (10)-(14) with initial condition and $s^{0}(c), c \in G_{d}(\sigma)$ is also the optimal trajectory. Let the point $\xi_{v} s \in G_{d}(\sigma)(v=1,2, \ldots, k)$ and any element $y(c) \in X$ be specified. If $x(\sigma)=y(c)$, then the state of the system in the point $\xi_{V} \sigma$ is determined by

$s\left(\xi_{v} \sigma\right)=\hat{F}_{v}(\sigma, \aleph, y, \omega(\sigma))$

We consider the following problem:

$$
\begin{aligned}
& \xi_{v} s(c)=\hat{F}_{v}(c, s(c), x(c), \omega(c)), c \in G_{d}\left(\xi_{v} \sigma\right) \\
& s\left(\xi_{v} \sigma\right)=\hat{F}_{v}(\sigma, \aleph, y) \\
& x(c) \in X(c), c \in G_{d}\left(\xi_{v} \sigma\right) \\
& \bar{J}(x)=M_{\omega}\left\{\phi\left(s\left(c^{L}\right)\right)\right\} \rightarrow \min
\end{aligned}
$$

If $\hat{y}(c), c \in G_{d}\left(\xi_{v} \sigma\right)$ and $\hat{s}(c), c \in G_{d}\left(\xi_{v} \sigma\right)$ are optimal control and optimal trajectory respectively, then 
International Journal on Cybernetics \& Informatics (IJCI) Vol. 3, No. 4, August 2014

$M_{\omega}\left\{\phi\left(s\left(c^{L}\right)\right)\right\}=B\left(\xi_{v} \sigma, \hat{F}_{v}(\sigma, \aleph, \hat{y}, \omega(\sigma))\right)$

can be found.

For (10)-(14), let $\tilde{x}(c)$ be an admissible control below.

$\tilde{x}(c)=\left\{\begin{array}{l}y \quad, c=\sigma \\ \hat{y}(c), c \in G_{d}\left(\xi_{v} \sigma\right)\end{array}\right.$

Also, $\tilde{s}(c)$ can be obtained by

$\tilde{s}(c)=\left\{\begin{array}{l}\hat{F}_{v}(\sigma, \aleph, y, \omega(\sigma)), c=\sigma \\ \hat{s}(c), c \in G_{d}\left(\xi_{v} \sigma\right)\end{array}\right\}$

It is evident that the value of $\bar{J}(x)=M_{\omega}\left\{\phi\left(s\left(c^{L}\right)\right)\right\}$ to control $\tilde{x}(c)$ is determined by

$$
M_{\omega}\left\{\phi\left(\widetilde{s}\left(c^{L}\right)\right)\right\}=M_{\omega}\left\{\phi\left(\hat{s}\left(c^{L}\right)\right)\right\}==B\left(\xi_{v} \sigma, \hat{F}_{v}(\sigma, \aleph, \hat{y}, \omega(\sigma))\right) .
$$

Since $\tilde{x}(c), c \in G_{d}(\sigma)$ is not largely optimal control, we can state

$$
M_{\omega}\left\{\phi\left(\tilde{s}\left(c^{L}\right)\right)\right\} \geq M_{\omega}\left\{\phi\left(s^{0}\left(c^{L}\right)\right)\right\}=B(\sigma, \aleph)
$$

Thus, we have

$$
B(\sigma, \aleph) \leq B\left(\xi_{v} \sigma, \hat{F}_{v}(\sigma, \aleph, \hat{y}, \omega(\sigma))\right)
$$

On the other hand, if $y(c)=x^{0}(\sigma)$, then by the principle of optimality [2],

$$
\hat{y}(c)\left(c \in G_{d}\left(\xi_{V} \sigma\right)\right)=x^{0}(c)\left(c \in G_{d}\left(\xi_{v} \sigma\right)\right)
$$

Therefore,

$$
B(\sigma, \aleph)=B\left(\xi_{v} \sigma, \hat{F}_{v}\left(\sigma, \aleph, x^{0}(\sigma), \omega(\sigma)\right)\right)
$$

By (34) and (35), Bellman equation[5] can be determined by

$$
\begin{aligned}
& B(\sigma, \aleph)=\min _{y \in X(\sigma)} B\left(\xi_{v} \sigma, \hat{F}_{v}(\sigma, \aleph, \hat{y}, \omega(\sigma))\right), \\
& \aleph \in S
\end{aligned}
$$


International Journal on Cybernetics \& Informatics (IJCI) Vol. 3, No. 4, August 2014

\section{Conclusions}

It is shown that Bellman equation for optimal processes with stochastic sequential machine is obtained and the principle of optimality is proven.

\section{REFERENCES}

[1] Anderson, J. A., (2004) Discrete Mathematics witk Combinatorics, Prentice Hall, New Jersey, p.45 "This is my paper", ABC Transactions on ECE, Vol. 10, No. 5, pp120-122.

[2] Boltyanskii, V. G., (1978) Optimal Control of Discrete Systems, John Willey, New York, p.363.

[3] Gaishun, I. V., (1983) Completely Solvable Multidimensional Differential Equations, Nauka and Tekhnika, Minsk, p.231.

[4] Hac1, Y., Ozen, K., (2009) “Terminal Control Problem for Processes Represented by Nonlinear Multi Binary Dynamic System”, Control and Cybernetics, Vol. 38, No. 3, pp625-633.

[5] Bellman, R., (1957) Dynamic Programming, Princeton University Press, Princeton, p.12

[6] Yermolyev, Y. M., (1976) Stochastic Programming Methods, Nauka (in Russian), p.240.

[7] Yablonsky, S. V., (1989) Introduction to Discrete Mathematics, Mir Publishers, Moscow, p.9.

[8] Fraileigh, J. B., (1998) A First Course in Abstract Algebra, 6th ed. Addison--Wesley Publisher, p.419-423

\section{Authors}

Yakup H. HACI received Ph.D at physics and mathematics in Azerbaijan NationalAcademy of Sciences at the Institute of Cybernetics. He is currently working as professor and head of department of Mathematics, in Canakkale Onsekiz Mart University. His primary research interests are in the areas of the theory of multiparametric binary sequential machines, linear and nonlinear optimization and discrete mathematics. He is an author(co-author) of over 38 papers.

Muhammet CANDAN received his M.Sc degree from Canakkale Onsekiz Mart University, Canakkale, Turkey, in 2012. He is currently Ph.D. candidate and working as a research assistant in Canakkale Onsekiz Mart University. His research interests cover applied mathematics, finite dynamical systems, graph theory. 